Supporting Information for:

\title{
A Vector-Selective Reaction Enables Efficient Construction of Specific Topology upon the Primary Side of $\beta$-Cyclodextrin
}

\author{
De-Qi Yuan,* Yumika Kitagawa, Makoto Fukudome, and Kahee Fujita* \\ Department of Molecular Medicinal Sciences, Graduate School of Biomedical Sciences, \\ Nagasaki University, Nagasaki 852-8521 (Japan) \\ deqiyuan@.nagasaki-u.ac.jp; fujita@.nagasaki-u.ac.jp
}

$\underline{\text { Contents }}$

1. Experimental

1-1. Syntheses of 2D and 2L S2

1-2. Syntheses of 3D and 3L S3

1-3. Enzymatic hydrolyses of 3D and 3L $\quad$ S4

1-4. Preparation of $\mathbf{5 D}$ and $\mathbf{5 L} \quad$ S4

2. Spectra

${ }^{1} \mathrm{H}$ and ${ }^{13} \mathrm{C}$ NMR spectra of 2D $\quad$ S6

${ }^{1} \mathrm{H}$ and ${ }^{13} \mathrm{C}$ NMR spectra of $\mathbf{2 L} \quad$ S8

${ }^{1} \mathrm{H}$ and ${ }^{13} \mathrm{C}$ NMR spectra of 3D $\quad \mathrm{S} 10$

${ }^{1} \mathrm{H}$ and ${ }^{13} \mathrm{C}$ NMR spectra of $\mathbf{3 L} \quad \mathrm{S} 12$

${ }^{1} \mathrm{H}$ and ${ }^{13} \mathrm{C}$ NMR spectra of 5D $\quad \mathrm{S} 14$

${ }^{1} \mathrm{H}-{ }^{1} \mathrm{H}$ COSY, ${ }^{1} \mathrm{H}-{ }^{13} \mathrm{C}$ HSQC and ${ }^{1} \mathrm{H}_{-}{ }^{13} \mathrm{C}$ HMBC spectra of 5D S16

$\begin{array}{ll}\text { PSD-MS spectrum of } \mathbf{5 L} & \text { S19 }\end{array}$

${ }^{1} \mathrm{H}$ and ${ }^{13} \mathrm{C}$ NMR spectra of $\mathbf{5 L} \quad$ S20

${ }^{1} \mathrm{H}-{ }^{1} \mathrm{H}$ COSY, ${ }^{1} \mathrm{H}-{ }^{13} \mathrm{C}$ HSQC and ${ }^{1} \mathrm{H}-{ }^{13} \mathrm{C}$ HMBC spectra of 5L $\mathbf{S} 22$

Fluorescence titration spectra of 2D, 2L, 3D and 3L

$\begin{array}{ll}\text { for the derivation of binding constants } & \text { S25 }\end{array}$ 


\section{Experimental}

\section{1-1. Syntheses of $\mathbf{2 D}$ and $\mathbf{2 L}$}

Synthesis of 2L: A solution containing compound 1 (413 mg), L-cysteine monohydrochloride $(250 \mathrm{mg})$ and $\mathrm{Na}_{2} \mathrm{CO}_{3}(268 \mathrm{mg})$ in $\mathrm{H}_{2} \mathrm{O}-\mathrm{DMF}(2: 1$ by volume, $6 \mathrm{~mL}$ ) was stirred at $90{ }^{\circ} \mathrm{C}$ for $1.5 \mathrm{~h}$. After neutralization, acetone was added and the precipitates were collected and chromatographed on a reversed-phase Lobar column (Rp-18, size C, 0\% - $40 \%$ aqueous ethanol as the gradient eluent) to give the $6^{\mathrm{A}}-(\mathrm{L}-\mathrm{cysteine})-6^{\mathrm{A}}-\mathrm{deoxy}-\beta-\mathrm{CD}$ (321 mg). The latter compound and $\mathrm{Na}_{2} \mathrm{CO}_{3}(167 \mathrm{mg})$ were dissolved in water $(5 \mathrm{~mL})$, and dansyl chloride $(200 \mathrm{mg}$ ) in acetonitrile solution $(5 \mathrm{~mL})$ was added. After being stirred at $\mathrm{rt}$. for $70 \mathrm{~min}$, the mixture was neutralized with $1 \mathrm{M} \mathrm{HCl}$, and added to acetone. The precipitates were collected and chromatographed (Rp-18 Lobar column, size C, 0\% - 30\% aqueous ethanol as the gradient eluent) to give compound $\mathbf{2 L}$ (195 mg, 41\%). TOF-MS: $\mathrm{m} / z$ $1494\left[\mathrm{M}+\mathrm{Na}^{+}\right], 1510\left[\mathrm{M}+\mathrm{K}^{+}\right] \cdot{ }^{1} \mathrm{H}-\mathrm{NMR}\left(\mathrm{D}_{2} \mathrm{O}-\mathrm{DMSO}-\mathrm{d}_{6}, \mathrm{CH}_{3} \mathrm{CN}\right.$ int.): $\delta 8.54$ (d, $J=8.3$ $\mathrm{Hz}, 1 \mathrm{H}), 8.27$ (d, $J=8.8 \mathrm{~Hz}, 1 \mathrm{H}), 8.19(\mathrm{~d}, J=7.3 \mathrm{~Hz}, 1 \mathrm{H}), 7.68$ (t, $J=$ ca. $8.0 \mathrm{~Hz}, 1 \mathrm{H})$, $7.54(\mathrm{t}, J=7.8 \mathrm{~Hz}, 1 \mathrm{H}), 7.35(\mathrm{~d}, J=7.3 \mathrm{~Hz}, 1 \mathrm{H}), 5.06-4.7$ (m, overlapped with HOD, 7H), $4.10-3.05(\mathrm{~m}, 39 \mathrm{H}), 2.86(\mathrm{~s}, 6 \mathrm{H}), 2.86-2.71(\mathrm{~m}, 3 \mathrm{H}), 2.34(\mathrm{~m}, 2 \mathrm{H}), 2.15(\mathrm{~m}, 1 \mathrm{H})$ ppm. ${ }^{13} \mathrm{C}-\mathrm{NMR}\left(\mathrm{D}_{2} \mathrm{O}, \mathrm{CH}_{3} \mathrm{CN}\right.$ int.): $\delta 175.2,137.1,129.9,129.6,129.5,128.9,128.7,125.8$, $124.5,123.3,119.9,118.2,103.0,102.8,102.5,102.0,85.5,82.0,81.9,81.7,81.5,81.3$, 74.3, 74.1, 74.0, 73.9, 73.3, 73.1, 72.9, 72.8, 72.8, 72.6, 72.5, 72.0, 71.6, 61.4, 61.1, 60.9, $60.8,60.5,60.3,56.8,47.4,47.1,35.1,33.1 \mathrm{ppm}$.

Synthesis of 2D: A procedure similar to that for the preparation of $2 \mathbf{L}$ was used. Yield: 43\% from 1. TOF-MS: $m / z 1494\left[\mathrm{M}+\mathrm{Na}^{+}\right], 1510\left[\mathrm{M}+\mathrm{K}^{+}\right] .{ }^{1} \mathrm{H}-\mathrm{NMR}\left(\mathrm{D}_{2} \mathrm{O}-\mathrm{DMSO}-\mathrm{d}_{6}\right.$, $\mathrm{CH}_{3} \mathrm{CN}$ int.): $\delta 8.52(\mathrm{~d}, J=8.3 \mathrm{~Hz}, 1 \mathrm{H}), 8.27(\mathrm{~d}, J=8.8 \mathrm{~Hz}, 1 \mathrm{H}), 8.18(\mathrm{~d}, J=7.3 \mathrm{~Hz}, 1 \mathrm{H})$, $7.69(\mathrm{t}, J=$ ca. $8.0 \mathrm{~Hz}, 1 \mathrm{H}), 7.52(\mathrm{t}, J=\mathrm{ca} .8 .0 \mathrm{~Hz}, 1 \mathrm{H}), 7.33(\mathrm{~d}, J=7.8 \mathrm{~Hz}, 1 \mathrm{H}), 5.06-4.7$ (m, overlapped with HOD, 7H), $4.10-2.95$ (m, $41 \mathrm{H}), 2.85(\mathrm{~s}, 6 \mathrm{H}), 2.75$ (dd, $J=13.2,4.4$ Hz, $1 \mathrm{H}), 2.46$ (br. d, $J=11.7 \mathrm{~Hz}, 1 \mathrm{H}), 2.33$ (dd, $J=13.2,9.3 \mathrm{~Hz}, 1 \mathrm{H}), 2.22$ (dd, $J=12.7$, 
$9.3 \mathrm{~Hz}, 1 \mathrm{H}$ ) ppm. ${ }^{13} \mathrm{C}-\mathrm{NMR}$ (DMSO-d 6 , TMS int.): $\delta 172.2,150.8,135.2,129.3,128.8$, $128.6,127.9,127.6,123.1,118.8,114.6,101.4,101.0,83.6,80.9,80.7,72.5,72.3,71.7$, $71.5,70.6,59.3,57.5,44.7,36.7,33.0 \mathrm{ppm}$.

\section{1-2. Syntheses of $\mathbf{3 D}$ and $\mathbf{3 L}$}

Synthesis of 3L: Compound 2L (472 mg), DCC (281 mg) and HOBt (47 mg) were dissolved in DMF (15 mL), and the resultant mixture was stirred at rt. for 1 day. The sugar species were precipitated with acetone and then applied to column chromatography (Rp-18, size C, eluted by a gradient of $20 \%-60 \%$ aqueous ethanol) to give the lactone $3 \mathrm{~L}$ (366 mg, 78.5\%). TOF-MS: $m / z 1475\left[\mathrm{M}+\mathrm{Na}^{+}\right], 1491\left(\mathrm{M}+\mathrm{K}^{+}\right] .{ }^{1} \mathrm{H}-\mathrm{NMR}\left(\mathrm{D}_{2} \mathrm{O}-\mathrm{DMSO}-\mathrm{d}_{6}, \mathrm{CH}_{3} \mathrm{CN}\right.$ int.): $\delta 8.54$ (d, $J=8.8 \mathrm{~Hz}, 1 \mathrm{H}), 8.22$ and 8.20 (two doublets, overlapping, $2 \mathrm{H}$ ), 7.68 and 7.66 (two triplets, overlapping, $2 \mathrm{H}), 7.40(\mathrm{~d}, J=7.8 \mathrm{~Hz}, 1 \mathrm{H}), 5.00-4.88$ (m, $7 \mathrm{H}), 3.92$ (br, $2 \mathrm{H}), 3.80-3.40$ (m, $38 \mathrm{H}), 3.29$ (d, J=12.2 Hz, $1 \mathrm{H}), 3.25-3.00$ (m, $4 \mathrm{H}), 2.83$ (s, 6 H) ppm. ${ }^{13} \mathrm{C}-\mathrm{NMR}: \delta 168.7,151.1,134.7,129.8,128.6,128.6,128.5,127.9,123.3,118.5$, $114.9,102.1,101.7,101.7,101.3,85.9,82.0,81.4,81.2,81.0,80.6,72.8,72.6,72.5,72.3$, $72.1,72.0,71.8,71.6,71.5,71.3,69.9,69.4,64.3,59.7,59.6,59.5,59.4,58.9,56.9,44.8$, 33.7, $32.2 \mathrm{ppm}$.

Syntheses of 3D: A procedure similar to that for the preparation of $3 \mathbf{L}$ was used. Yield: 81\%. TOF-MS: $m / z 1475\left[\mathrm{M}+\mathrm{Na}^{+}\right] .{ }^{1} \mathrm{H}-\mathrm{NMR}\left(\mathrm{D}_{2} \mathrm{O}-\mathrm{DMSO}-\mathrm{d}_{6}, \mathrm{CH}_{3} \mathrm{CN}\right.$ int.): $\delta 8.48$ (d, $J=$ $8.3 \mathrm{~Hz}, 1 \mathrm{H}$ ), 8.17 and 8.15 ( two doublets, overlapping, 2H), 7.67 (t, $J=8.0 \mathrm{~Hz}, 1 \mathrm{H}$ ), 7.57 (t, $J=7.8 \mathrm{~Hz}, 1 \mathrm{H}), 7.34(\mathrm{~d}, J=7.3 \mathrm{~Hz}, 1 \mathrm{H}), 5.03-4.87$ (m, $7 \mathrm{H}), 4.43$ (br, $1 \mathrm{H}), 3.95-$ 3.40 (m, $38 \mathrm{H}), 3.27$ (t, $J=9.3 \mathrm{~Hz}, 1 \mathrm{H}), 3.18$ (t, $J=9.3 \mathrm{~Hz}, 1 \mathrm{H}), 2.97$ (br, $2 \mathrm{H}), 2.81$ (s, 6

H), 2.65 (br. 2 H) ppm. ${ }^{13} \mathrm{C}-\mathrm{NMR}$ (DMSO-d 6 , TMS int.): $\delta 169.1,150.9,135.4,129.5$, $128.5,128.4,127.9,127.7,123.1,118.4,114.9,102.0,101.6,101.3,101.0,85.8,81.9,81.0$, $80.9,80.8,80.1,72.5,72.4,72.2,71.7,71.4,70.2,69.0,64.7,59.4,58.6,56.3,44.6,33.9$, $33.7 \mathrm{ppm}$ 
1-3. Enzymatic hydrolyses of 3D and 3L

General procedure: A mixture of $3(120 \mathrm{mg})$ and $\alpha$-amylase (EC 3.2.1.1 from Aspergillus oryzae, Sigma) (120 mg) in $0.2 \mathrm{M}$ acetate buffer (pH 5.5, $10 \mathrm{~mL}$ ) containing $0.01 \mathrm{M} \mathrm{CaCl}_{2}$ was allowed to stand for $84 \mathrm{~h}$ at $40{ }^{\circ} \mathrm{C}$. After being neutralized with $\mathrm{NaOH}$ solution and heated for $10 \mathrm{~min}$ in a boiling water-bath, the mixture was diluted to $500 \mathrm{~mL}$ with water, membrane-filtered (cellulose acetate, $0.8 \mu \mathrm{m}$ ), and chromatographed on a reversed-phase Lobar column (Rp-18, size C, eluted with a gradient of $0 \%-70 \%$ aqueous EtOH) to afford a major product 4.

4D: Yield 74\%. $R_{\mathrm{f}}=0.60\left(n-\mathrm{PrOH} / \mathrm{AcOEt} / \mathrm{H}_{2} \mathrm{O}=7 / 7 / 5\right)$. TOF-MS: $m / z 845\left[\mathrm{M}+\mathrm{Na}^{+}\right]$.

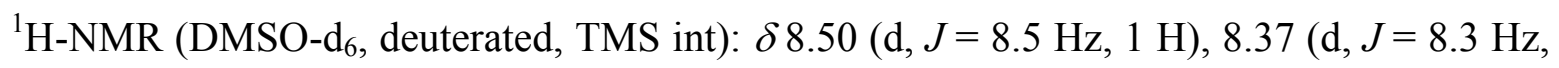
$1 \mathrm{H}), 8.16(\mathrm{~d}, J=7.2 \mathrm{~Hz}, 1 \mathrm{H}), 7.68(\mathrm{~m}, 2 \mathrm{H}), 7.43(\mathrm{~d}, J=7.2 \mathrm{~Hz}, 1 \mathrm{H}), 5.05-4.80(\mathrm{~m}, 2$ H), $4.40-2.50$ (m, overlapped with HOD and DMSO, $28 \mathrm{H}$ ) ppm.

4L: Yield 69\%. $R_{\mathrm{f}}=0.60\left(n-\mathrm{PrOH} / \mathrm{AcOEt} / \mathrm{H}_{2} \mathrm{O}=7 / 7 / 5\right)$. TOF-MS: $m / z 845\left[\mathrm{M}+\mathrm{Na}^{+}\right]$

\section{1-4. Preparation of $\mathbf{5 D}$ and $\mathbf{5} \mathbf{L}$}

Preparation of 5D: Compound 4D (50 mg) and $\mathrm{NaBH}_{4}(120 \mathrm{mg})$ were dissolved in water $(12 \mathrm{~mL})$ and the resultant mixture was stirred at r.t. for $1 \mathrm{~h}$. The reaction solution was acidified with $1 \mathrm{M} \mathrm{HCl}$, diluted with $20 \%$ ethanol $(500 \mathrm{~mL})$, membrane filtered and chromatographed on a reversed-phase Lobar column (Rp-18, Size C). Elution of the column with a gradient of $20 \%-70 \%$ aqueous ethanol afforded compound 5D (27.3 mg, 54\%). TOF-MS: $m / z 851\left[\mathrm{M}+\mathrm{Na}^{+}\right]$. PSD-MS: $m / z 851$ (100\%) $\left[\mathrm{M}+\mathrm{Na}^{+}\right], 689$ (40\%) [M $\left.162(\mathrm{Glc})+\mathrm{Na}^{+}\right], 669(12 \%)\left[\mathrm{M}-182+\mathrm{Na}^{+}\right], 507(25 \%)\left[\mathrm{M}-162-182+\mathrm{Na}^{+}\right] .{ }^{1} \mathrm{H} \mathrm{NMR}$ (DMSO-d 6 , TMS int.): $\delta 8.47(\mathrm{~d}, J=8.5 \mathrm{~Hz}, 1 \mathrm{H}), 8.27(\mathrm{~d}, J=8.5 \mathrm{~Hz}, 1 \mathrm{H}), 8.10(\mathrm{~d}, J=7.3$ $\mathrm{Hz}, 1 \mathrm{H}), 7.61(\mathrm{~m}, 2 \mathrm{H})$, and $7.26(\mathrm{~d}, J=7.3 \mathrm{~Hz}, 1 \mathrm{H})(\mathrm{Ar}-\mathrm{H}) ; 4.97\left(\mathrm{~d}, J=3.9 \mathrm{~Hz}, 1 \mathrm{H} ; 1^{\mathrm{B}}\right)$, 
$4.76\left(\mathrm{~d}, J=3.9 \mathrm{~Hz}, 1 \mathrm{H} ; 1^{\mathrm{A}}\right), 3.90-3.20(\mathrm{~m}, 18 \mathrm{H}), 3.11\left(\mathrm{t}, J=9.4 \mathrm{~Hz}, 1 \mathrm{H} ; 4^{\mathrm{B}}\right), 2.84(\mathrm{~s}, 6$ $\left.\mathrm{H} ; \mathrm{N}\left(\mathrm{CH}_{3}\right)_{2}\right), 2.74-2.64(\mathrm{~m}, 2 \mathrm{H})$, and $2.45-2.40(\mathrm{~m}, 2 \mathrm{H}) \mathrm{ppm} .{ }^{13} \mathrm{C} \mathrm{NMR}\left(\mathrm{DMSO}_{6}\right.$, TMS int.): $\delta 151.2,136.4,129.5,129.0,128.9,128.4,127.8,123.4,119.1$ and 114.9 (Ar); $100.8\left(1^{\mathrm{B}}\right), 100.2\left(1^{\mathrm{A}}\right), 83.2\left(4^{\mathrm{G}}\right), 82.3\left(4^{\mathrm{A}}\right), 73.2$ and $72.9\left(5^{\mathrm{B}}, 3^{\mathrm{B}}\right), 72.3$ and $72.1\left(2^{\mathrm{A}}, 3^{\mathrm{A}}\right)$, $72.3,71.1$ and $69.9\left(2^{\mathrm{G}}, 3^{\mathrm{G}} 5^{\mathrm{G}}\right), 71.2\left(2^{\mathrm{B}}\right), 70.2\left(5^{\mathrm{A}}\right), 69.4\left(4^{\mathrm{B}}\right), 62.5$ and $62.4\left(1^{\mathrm{G}}, 6^{\mathrm{G}}\right), 61.9$ (cys- $\alpha), 60.4\left(6^{\mathrm{B}}\right), 55.0($ cys- $\beta), 45.0\left(\mathrm{CH}_{3}\right), 34.3($ cys- $\gamma), 33.8\left(6^{\mathrm{A}}\right) \mathrm{ppm}$.

Preparation of 5L: A procedure similar to that for the preparation of 5D was used. Yield: 33 \%. TOF-MS: $m / z 851\left[\mathrm{M}+\mathrm{Na}^{+}\right]$. PSD-MS: $m / z 851$ (100\%) [M+ $\left.\mathrm{Na}^{+}\right], 689(40 \%)[\mathrm{M}-$ 162(Glc) $\left.+\mathrm{Na}^{+}\right], 669(12 \%)\left[\mathrm{M}-182+\mathrm{Na}^{+}\right] .{ }^{1} \mathrm{H}$ NMR (DMSO-d 6 , TMS int.): $\delta 8.47(\mathrm{~d}, J$ $=8.5 \mathrm{~Hz}, 1 \mathrm{H}), 8.28(\mathrm{~d}, J=8.7 \mathrm{~Hz}, 1 \mathrm{H}), 8.21(\mathrm{dd}, J=7.3,1.4 \mathrm{~Hz}, 1 \mathrm{H}), 7.61(\mathrm{~m}, 2 \mathrm{H})$, and $7.26(\mathrm{~d}, J=7.5 \mathrm{~Hz}, 1 \mathrm{H})(\mathrm{Ar}-\mathrm{H}) ; 4.93\left(\mathrm{~d}, J=3.9 \mathrm{~Hz}, 1 \mathrm{H} ; 1^{\mathrm{B}}\right), 4.82\left(\mathrm{~d}, J=3.9 \mathrm{~Hz}, 1 \mathrm{H} ; 1^{\mathrm{A}}\right)$, $3.90-3.20(\mathrm{~m}, 18 \mathrm{H}), 3.12\left(\mathrm{t}, J=9.2 \mathrm{~Hz}, 1 \mathrm{H} ; 4^{\mathrm{B}}\right), 2.84\left(\mathrm{~s}, 6 \mathrm{H} ; \mathrm{N}\left(\mathrm{CH}_{3}\right)_{2}\right), 2.80(\mathrm{dd}, J=$ 11.4, $\left.2.5 \mathrm{~Hz}, 1 \mathrm{H} ; 6^{\mathrm{A}}\right), 2.71\left(\mathrm{dd}, J=13.3,5.7 \mathrm{~Hz}, 1 \mathrm{H}\right.$; cys- $\gamma$ ), and $2.57-2.48\left(\mathrm{~m}, 2 \mathrm{H} ; 6^{\mathrm{A}^{\prime}}\right.$ and cys- $\left.\gamma^{\prime}\right)$ ppm. ${ }^{13} \mathrm{C}$ NMR (DMSO-d 6 , deuterated, TMS int.): $\delta 151.3,136.7,129.5,129.0$, 128.9, 128.2, 127.8, 123.4, 119.1 and $115.0(\mathrm{Ar})$; $100.9\left(1^{\mathrm{B}}\right), 100.2\left(1^{\mathrm{A}}\right), 83.0\left(4^{\mathrm{G}}\right), 82.4$ $\left(4^{\mathrm{A}}\right), 73.2$ and $72.9\left(5^{\mathrm{B}}, 3^{\mathrm{B}}\right), 72.4,72.3,72.2,71.3,71.1,70.3,69.9,69.5\left(4^{\mathrm{B}}\right), 62.5$ and 62.4 $\left(1^{\mathrm{G}}, 6^{\mathrm{G}}\right), 62.0($ cys- $\alpha), 60.5\left(6^{\mathrm{B}}\right), 55.3($ cys- $\beta), 45.0\left(\mathrm{CH}_{3}\right), 34.7($ cys- $\gamma), 34.0\left(6^{\mathrm{A}}\right)$ ppm. 


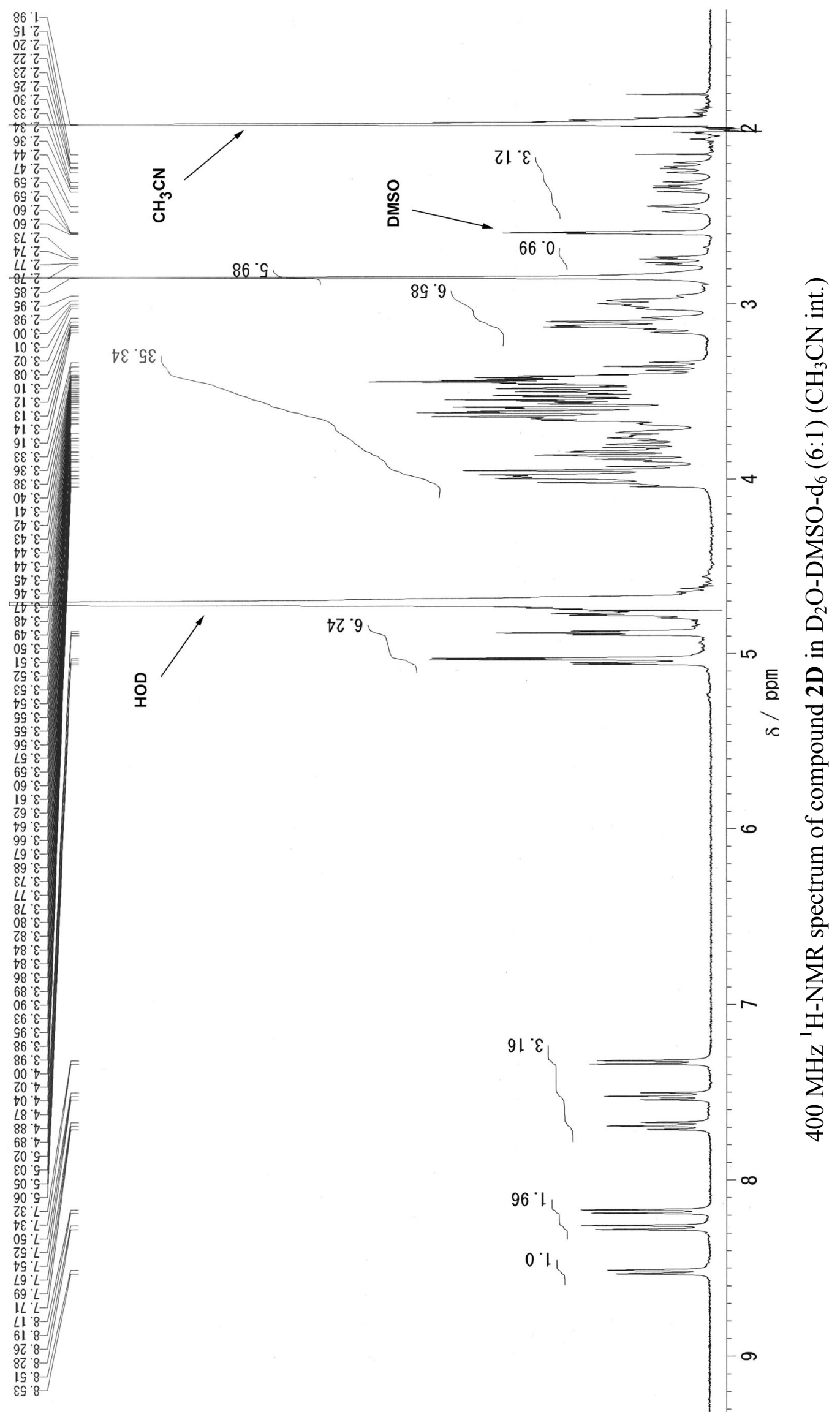




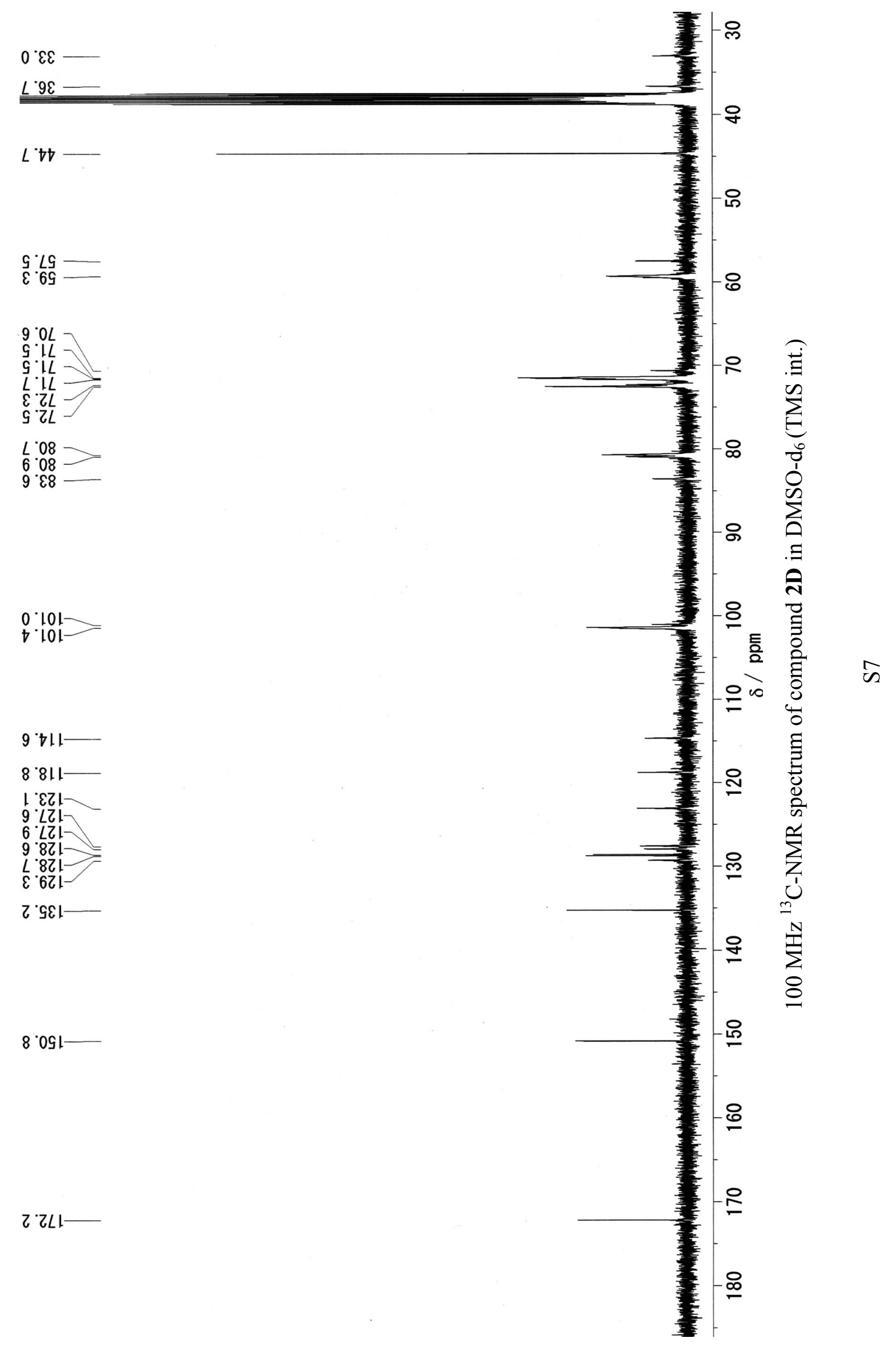




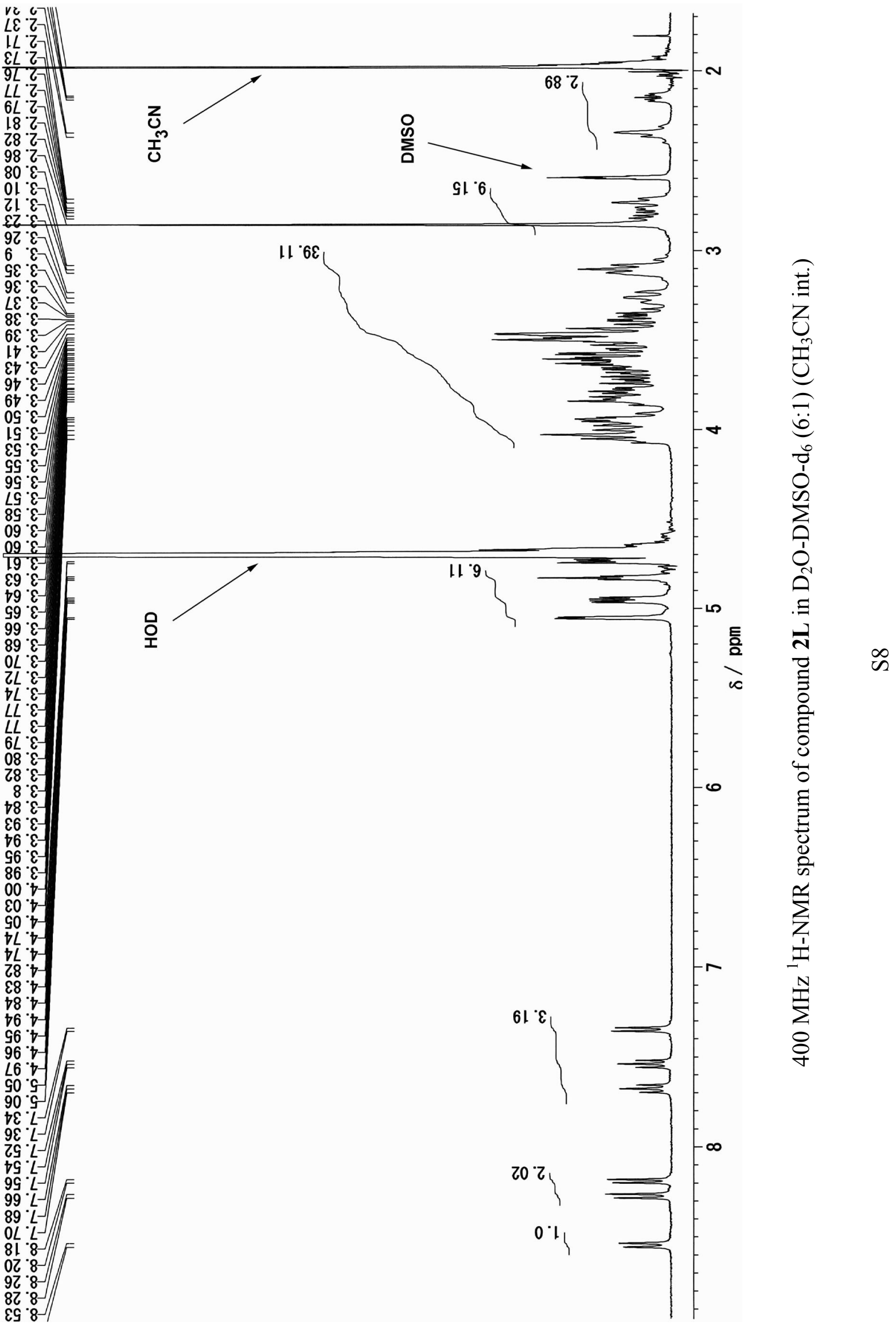



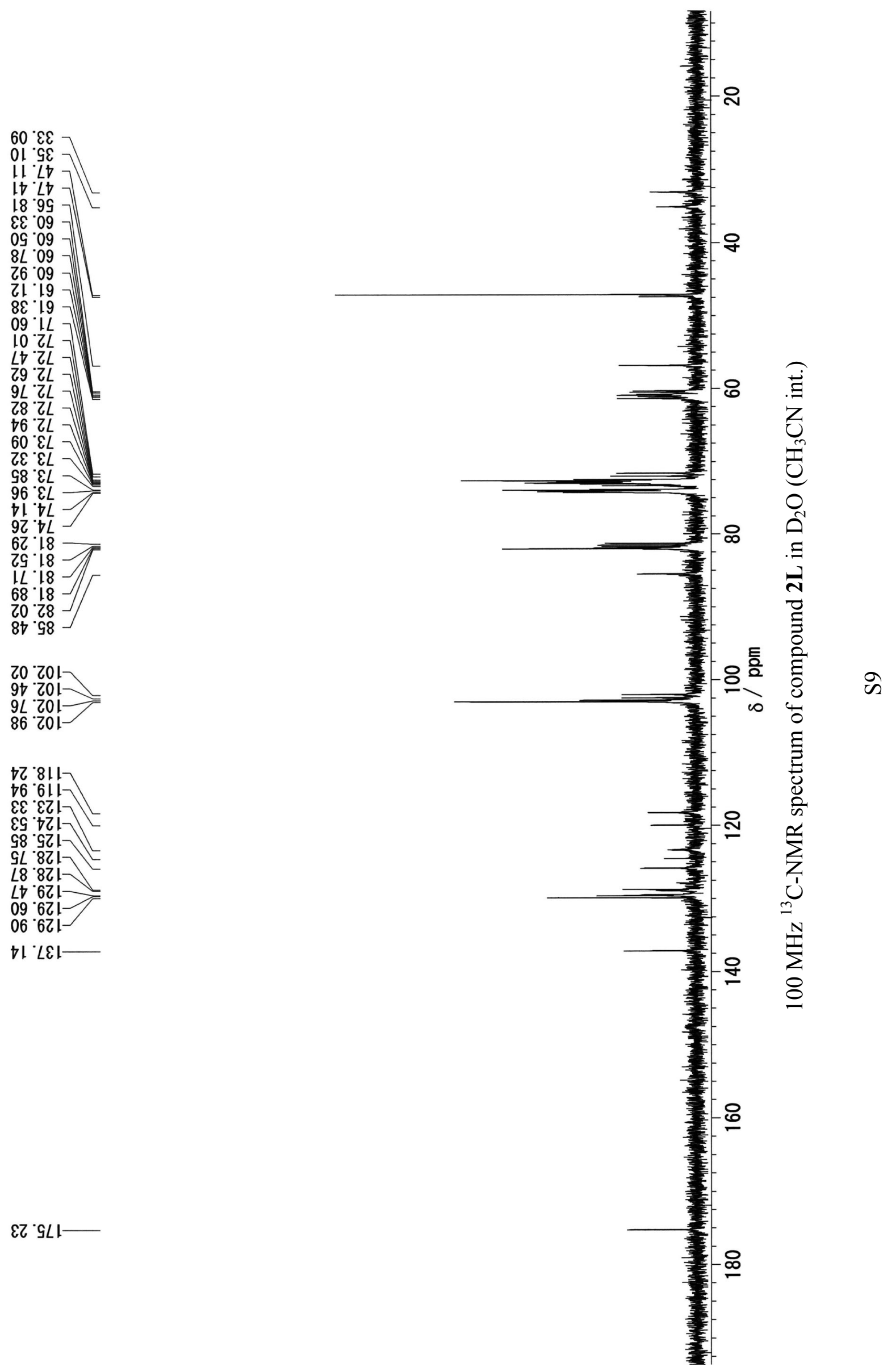


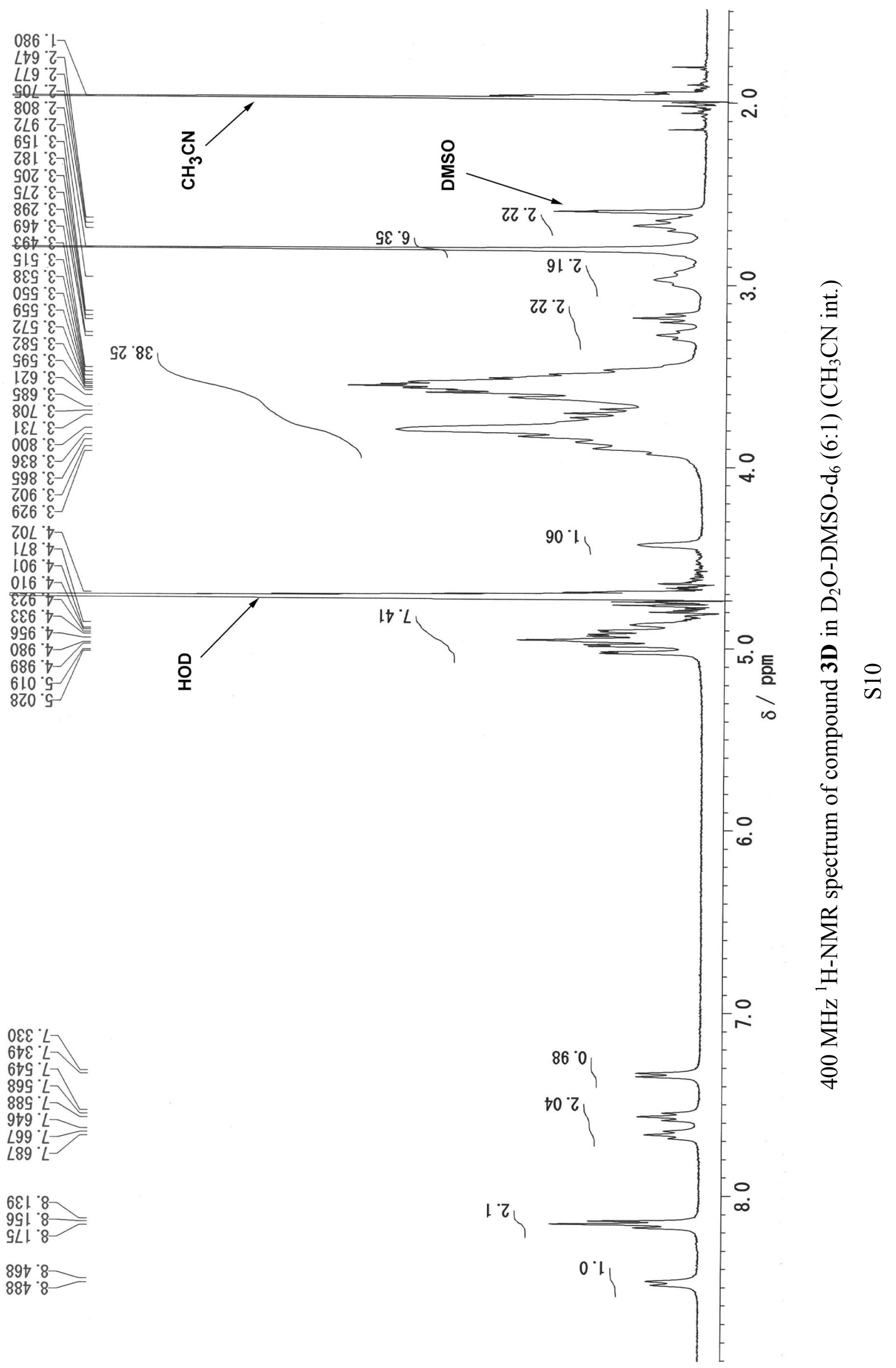




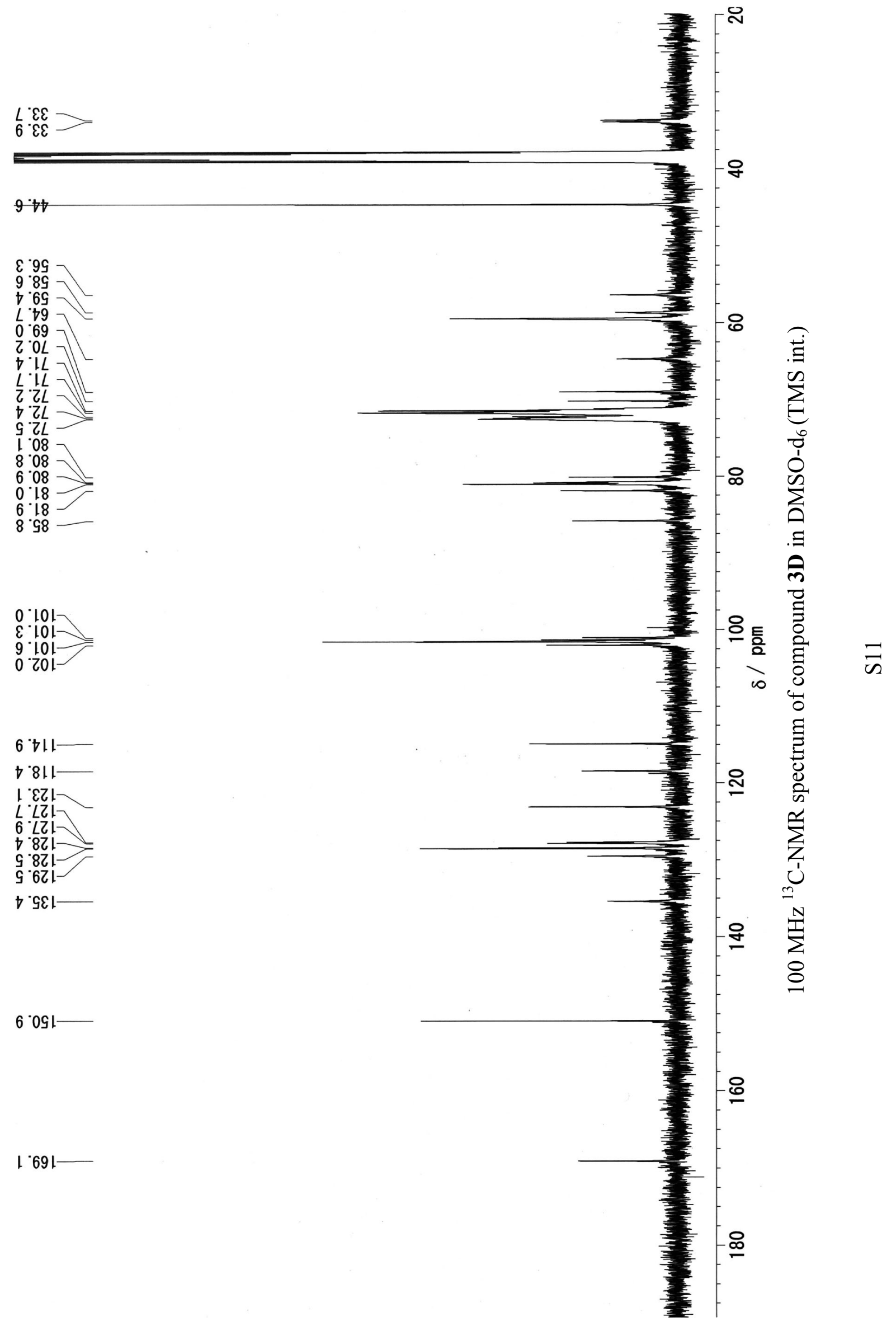




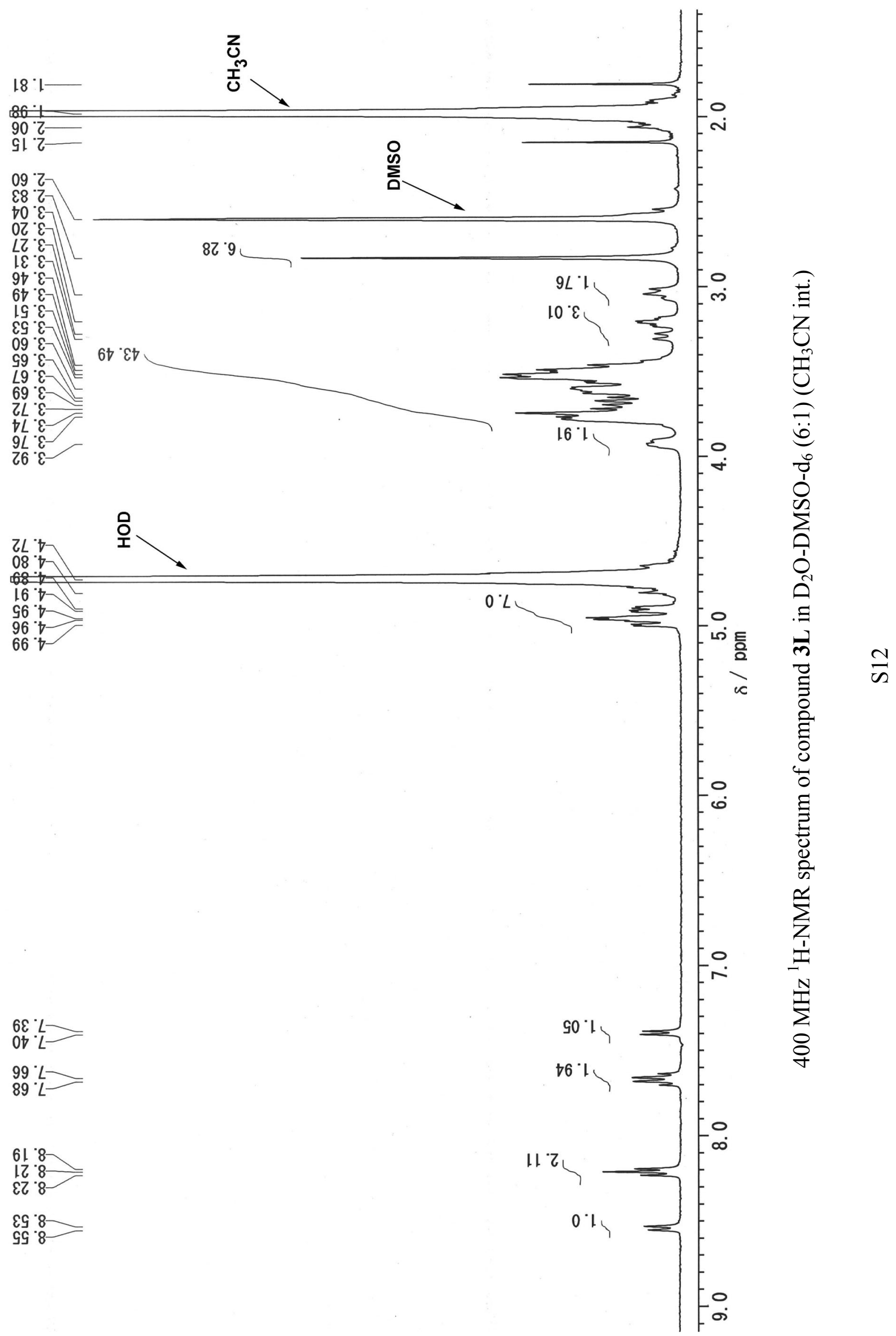




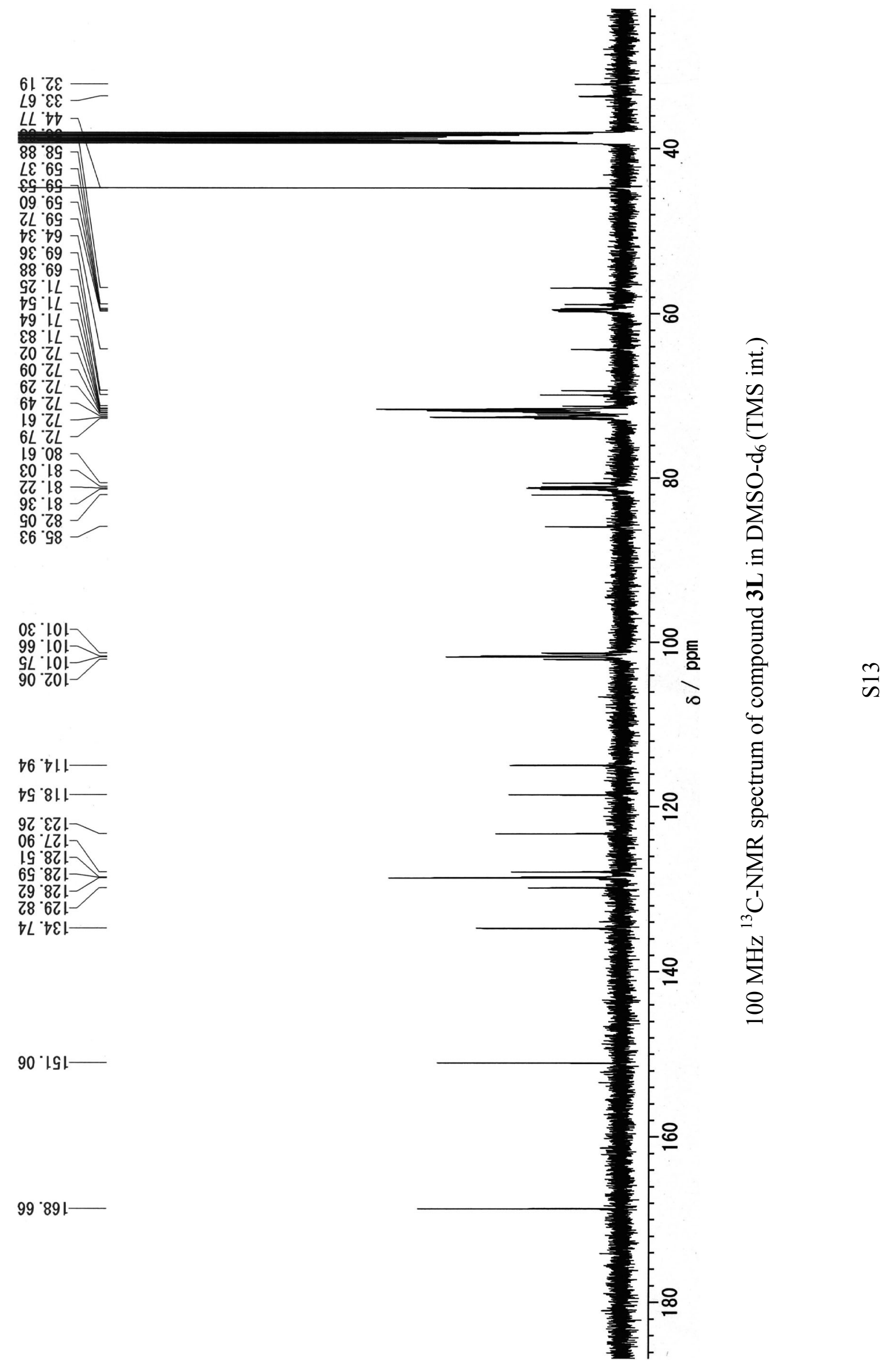




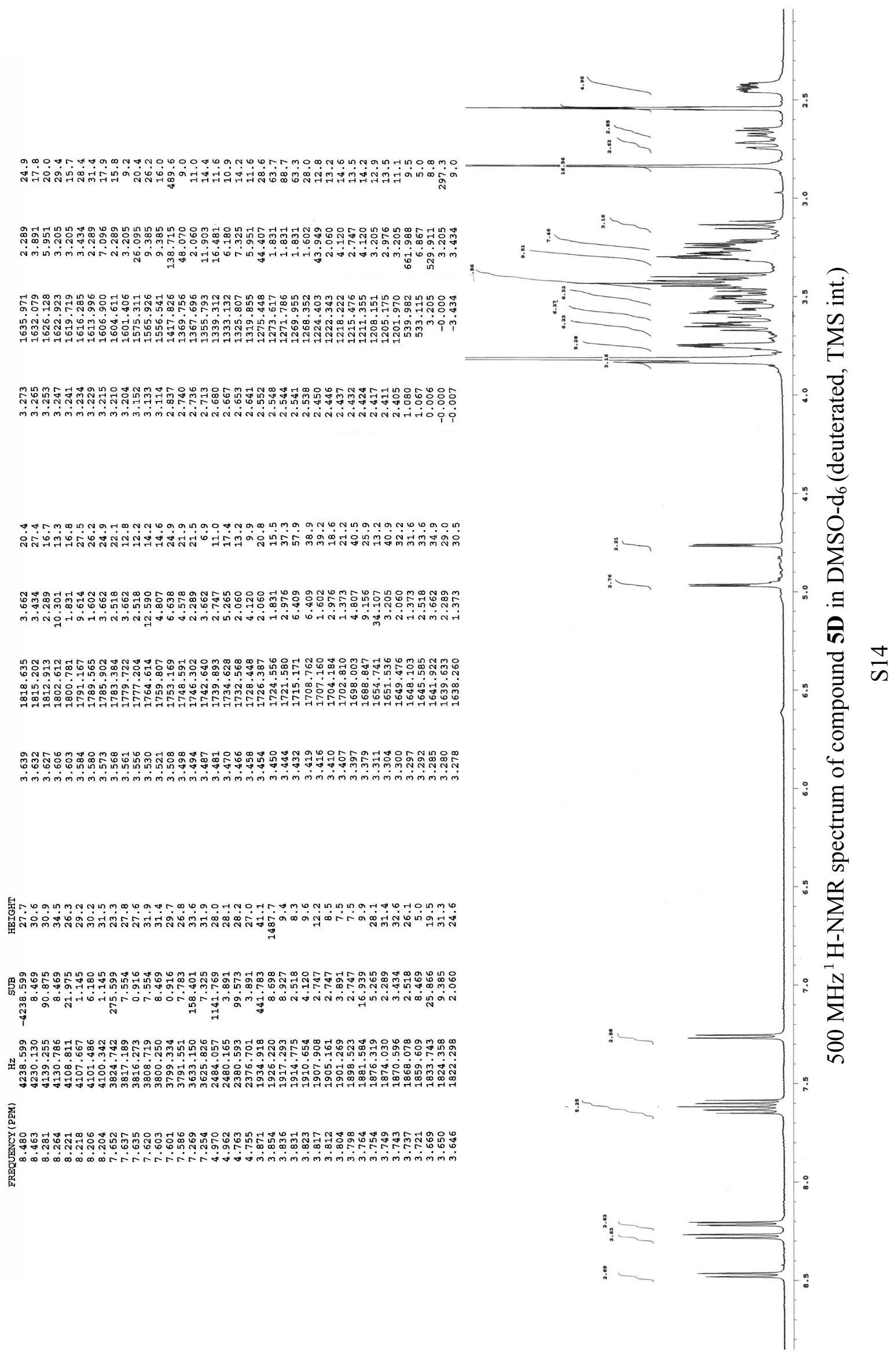




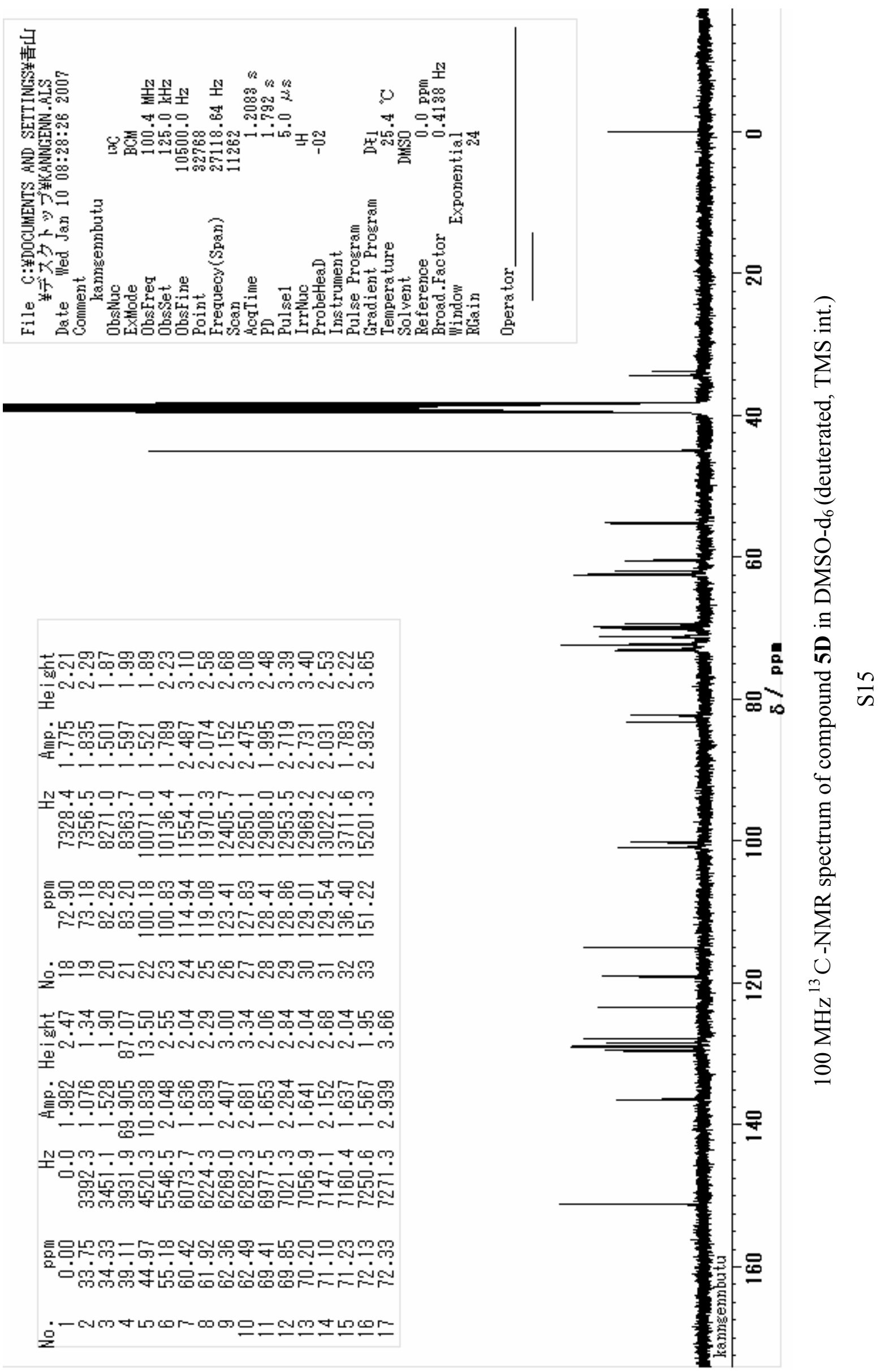




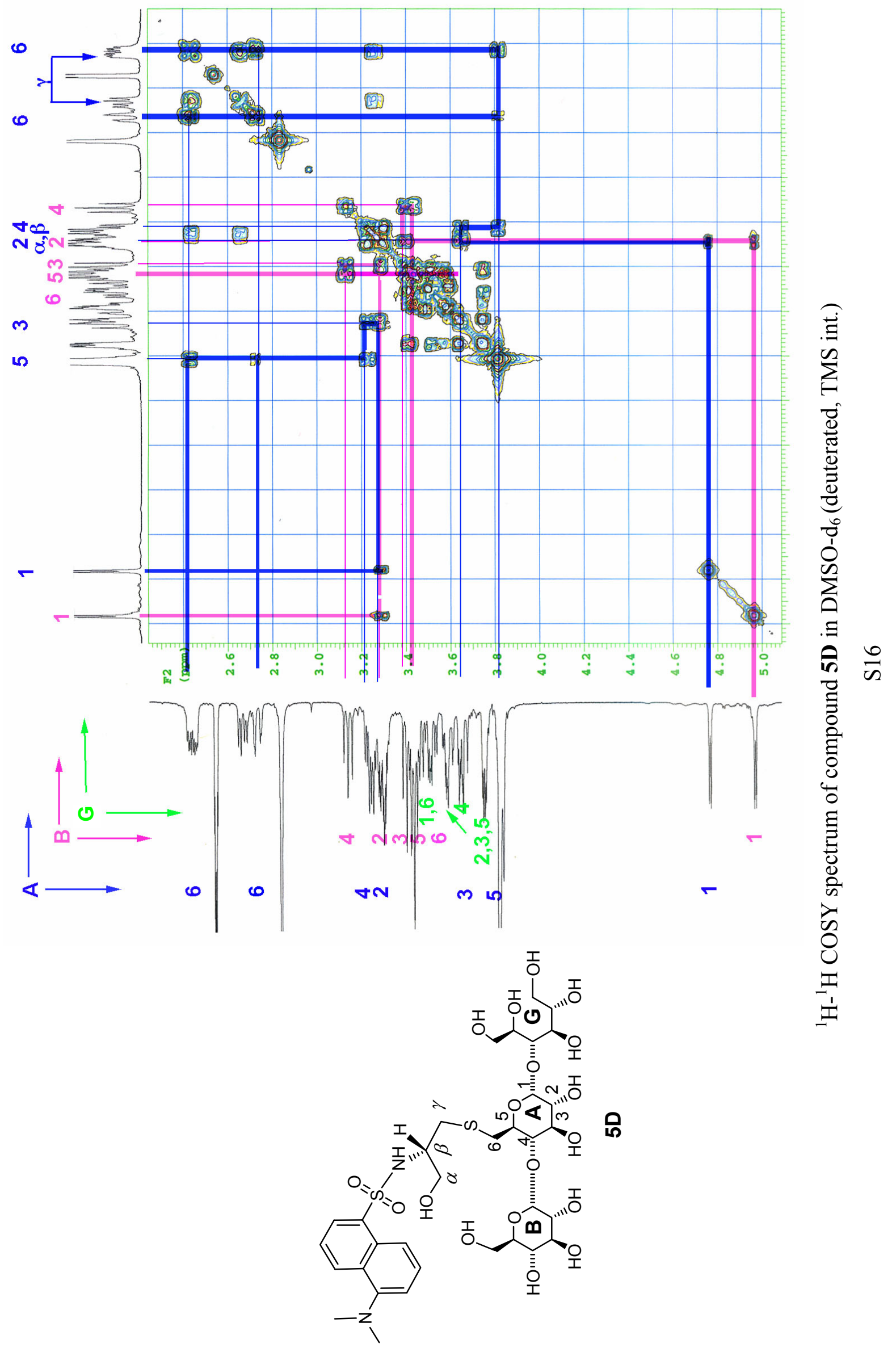




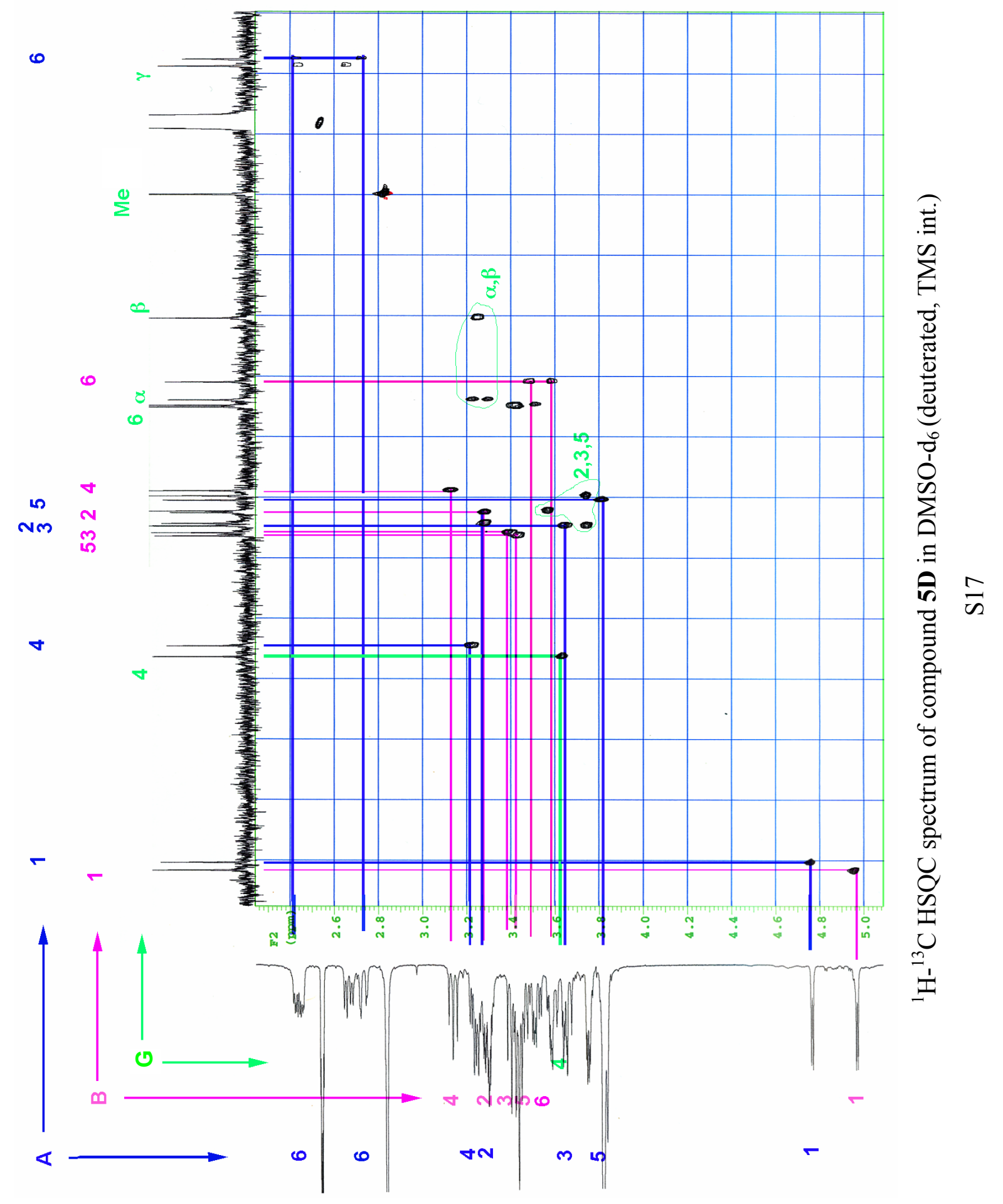




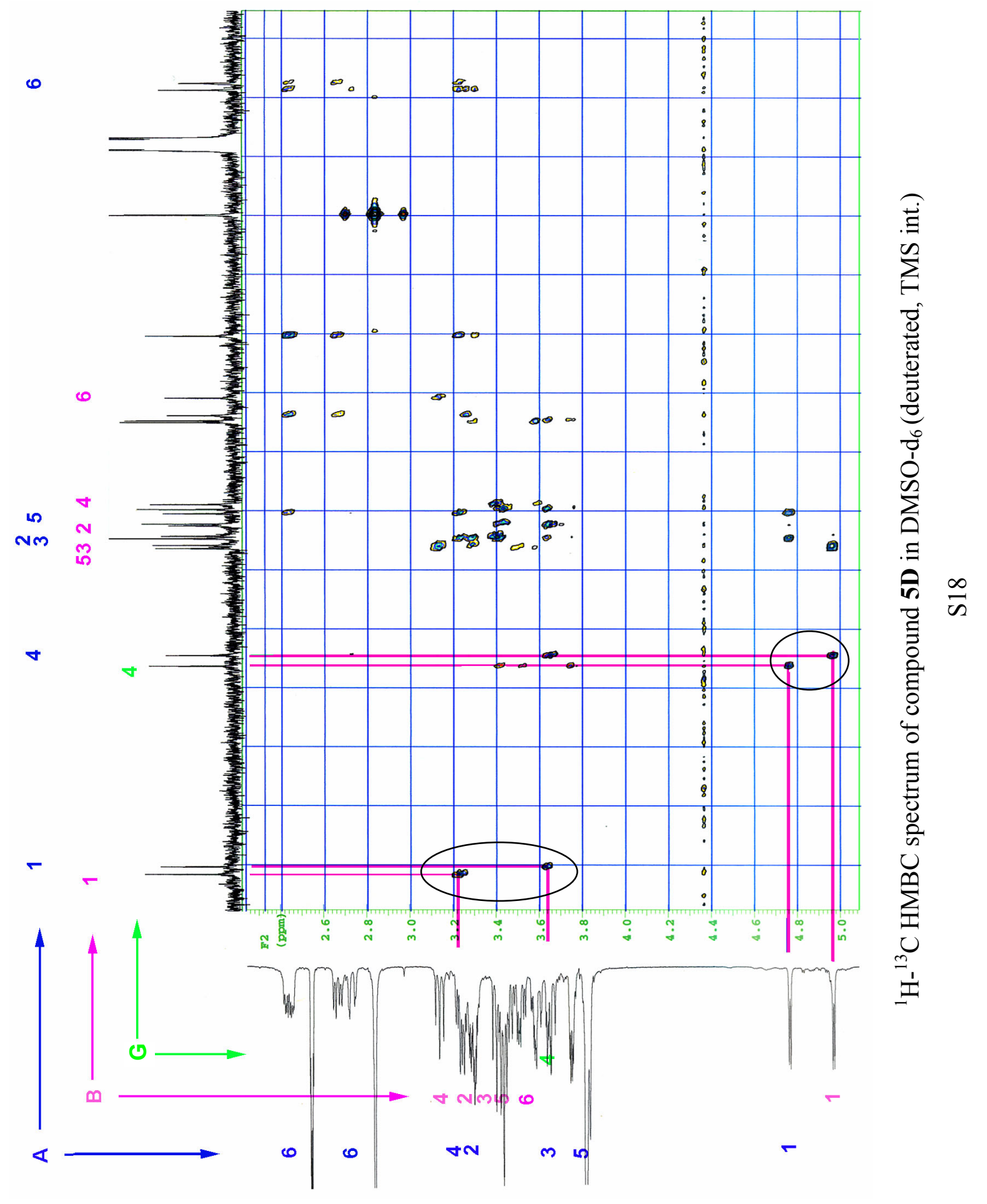




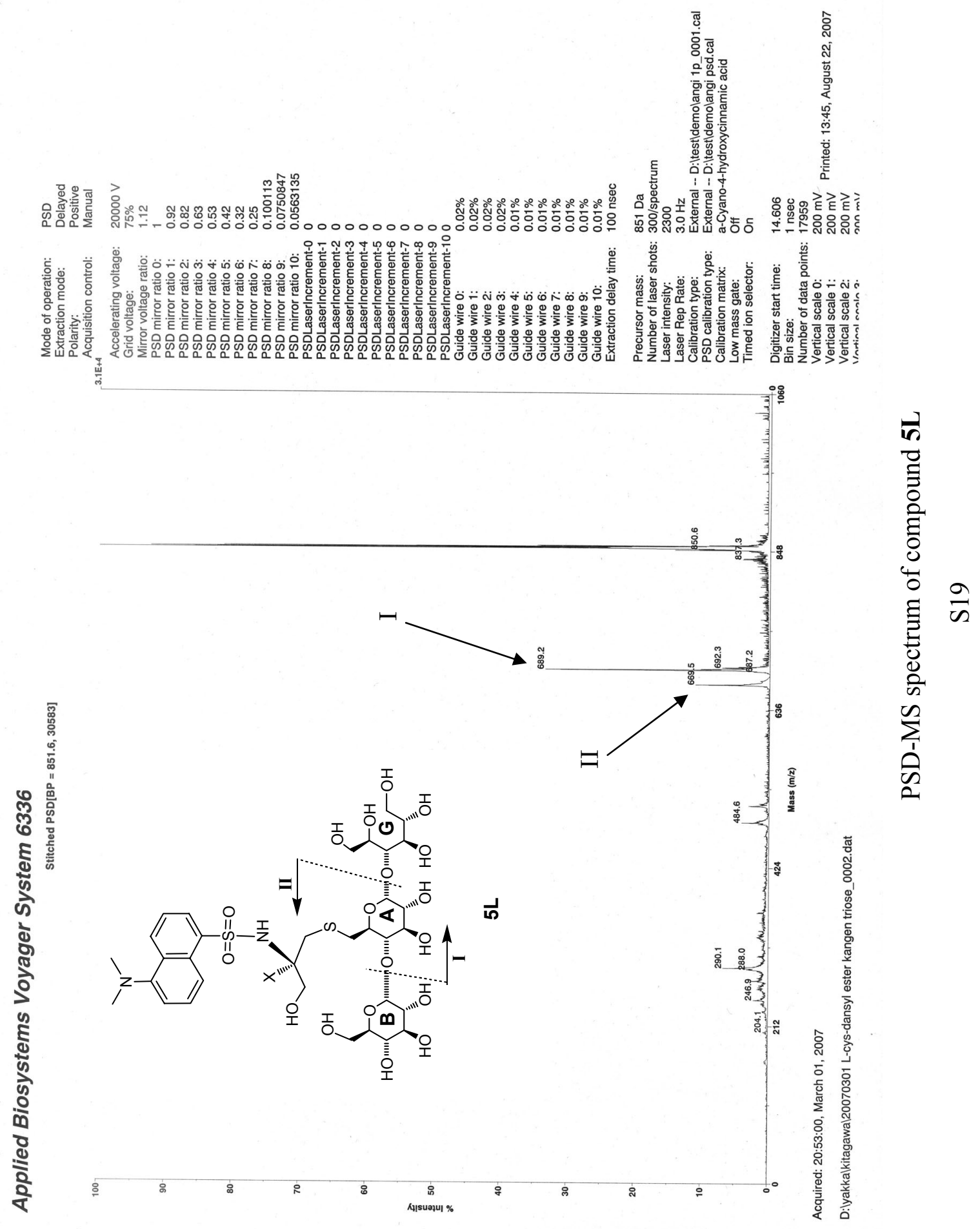




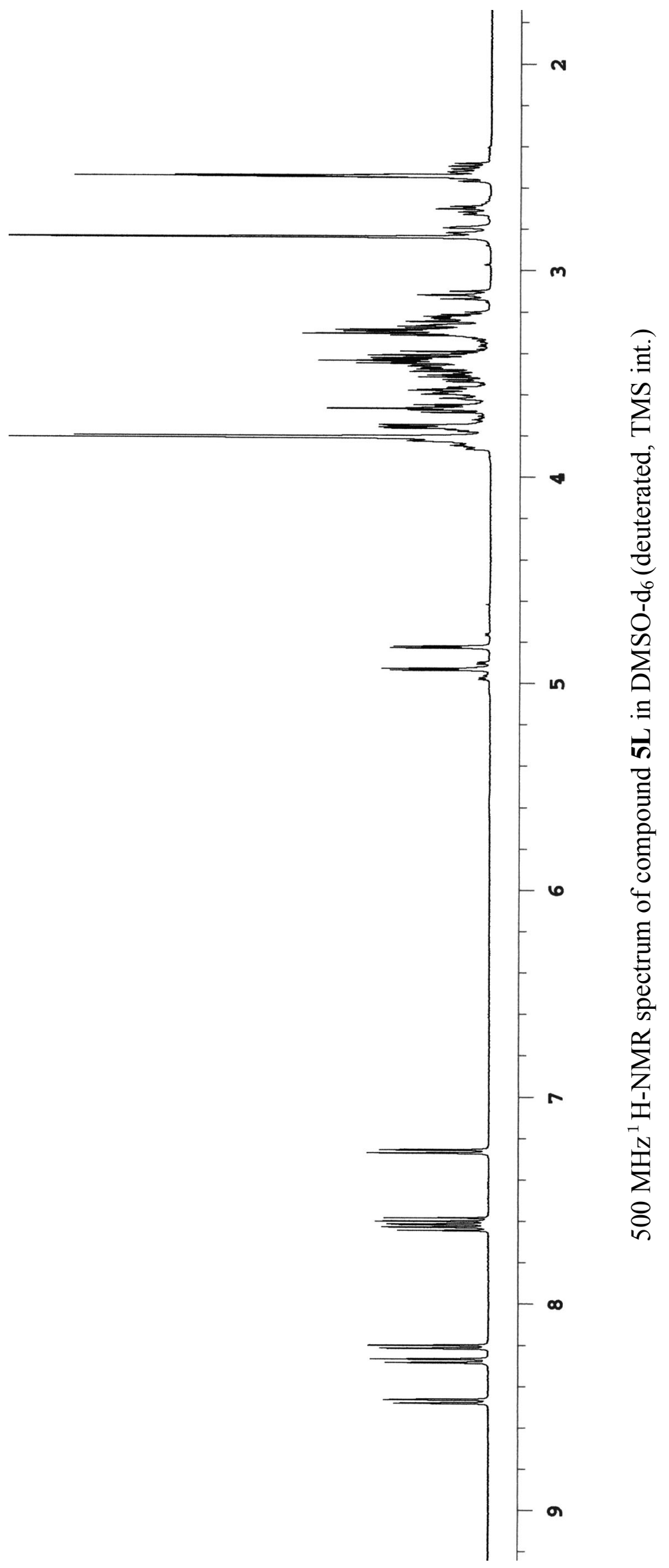

ถิ 


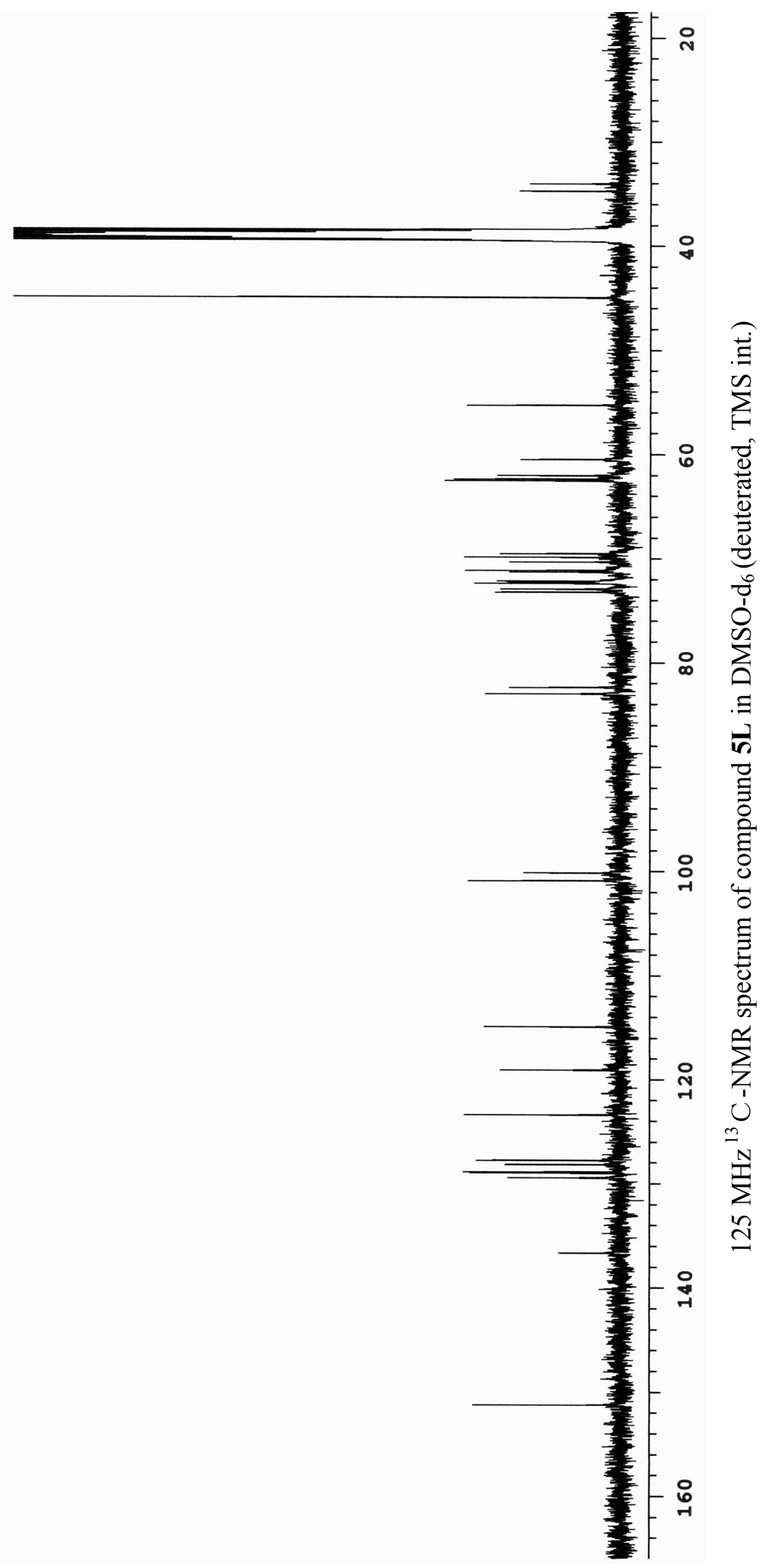




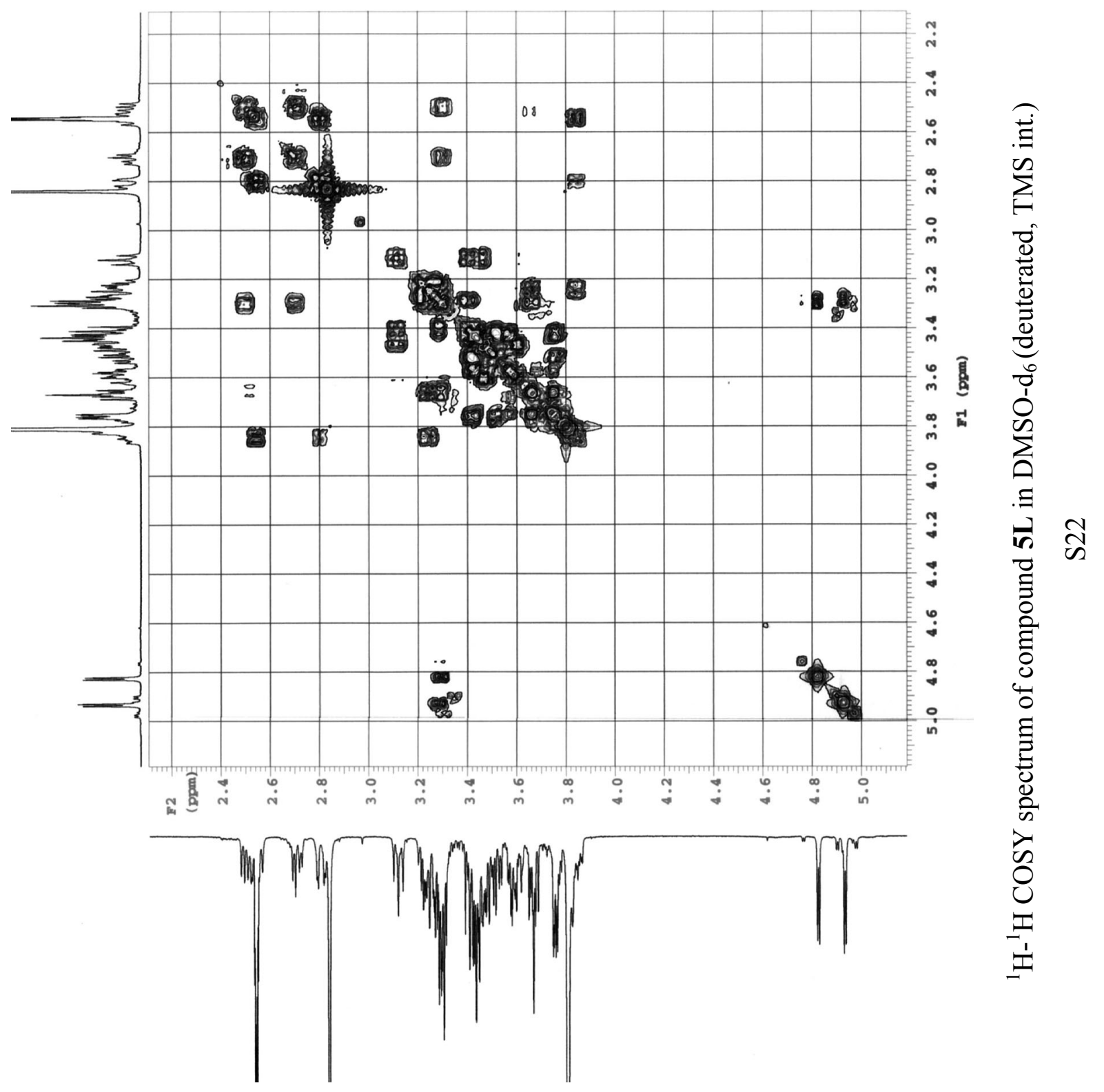




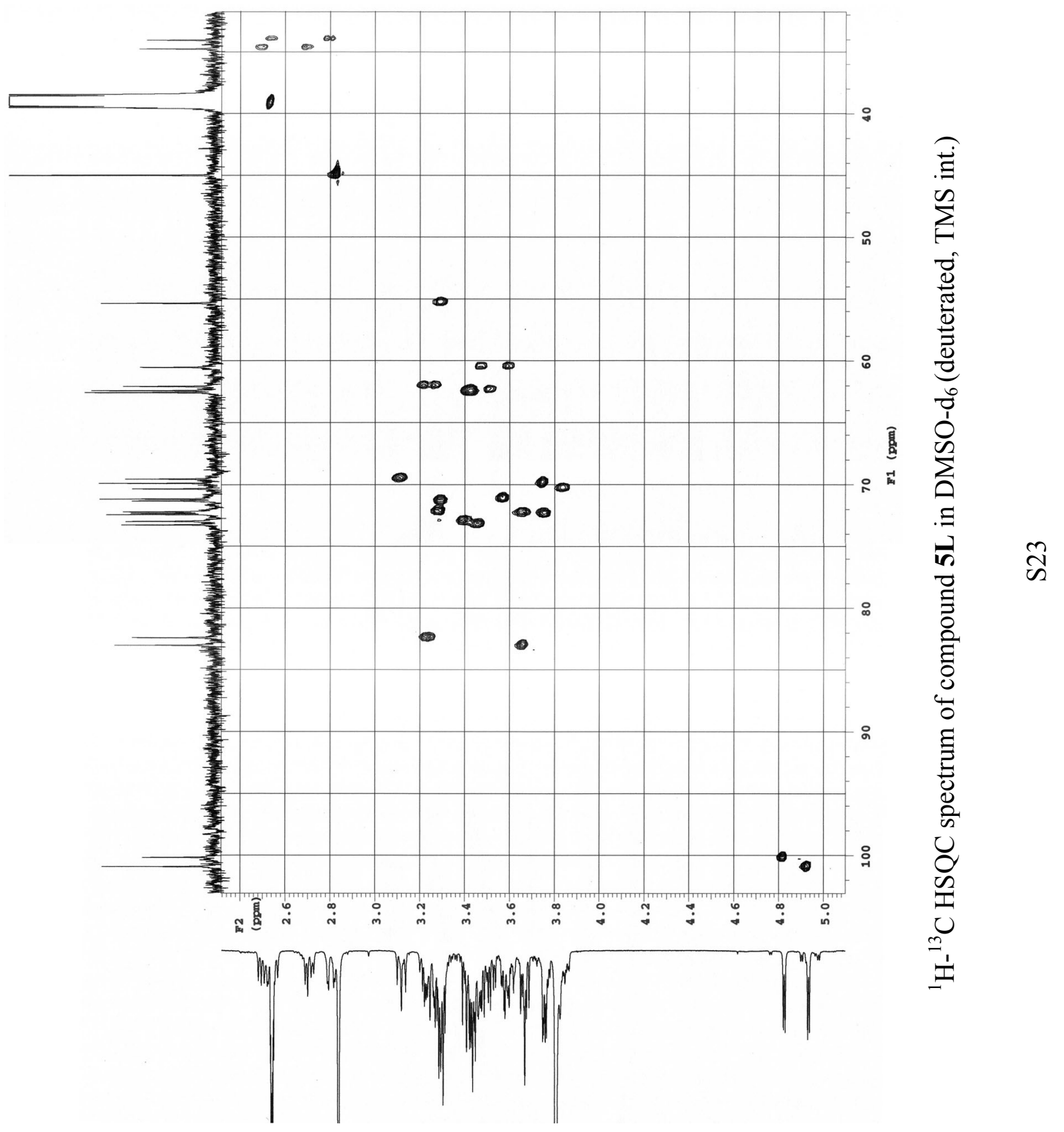




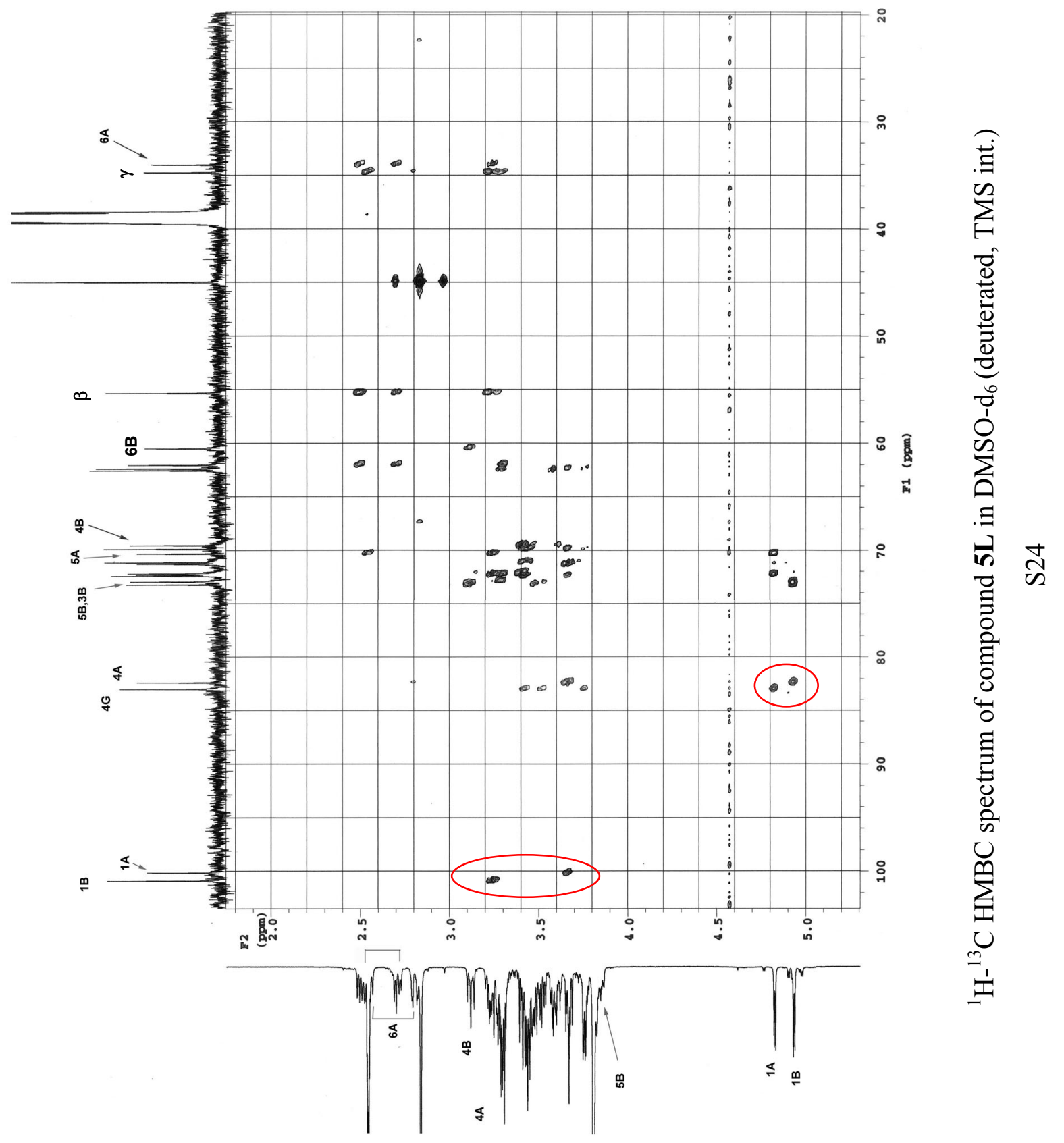




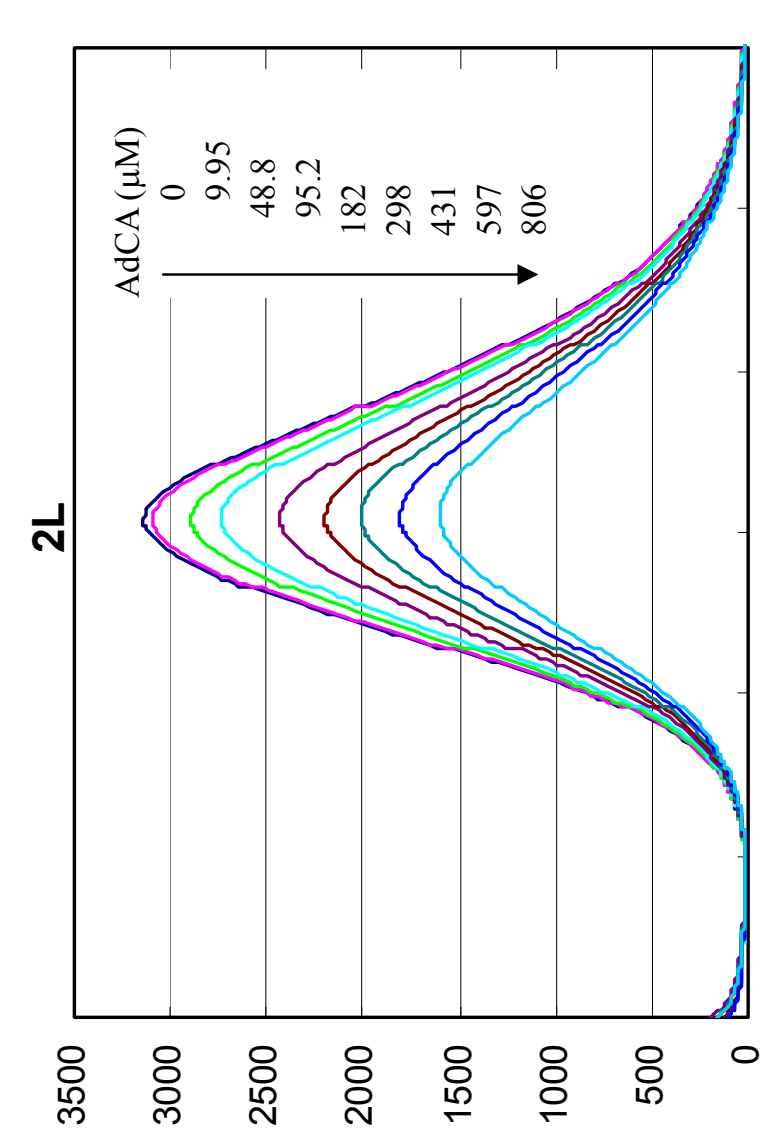

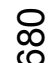

8
0

品

ถิ

을

กூ

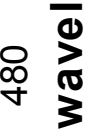

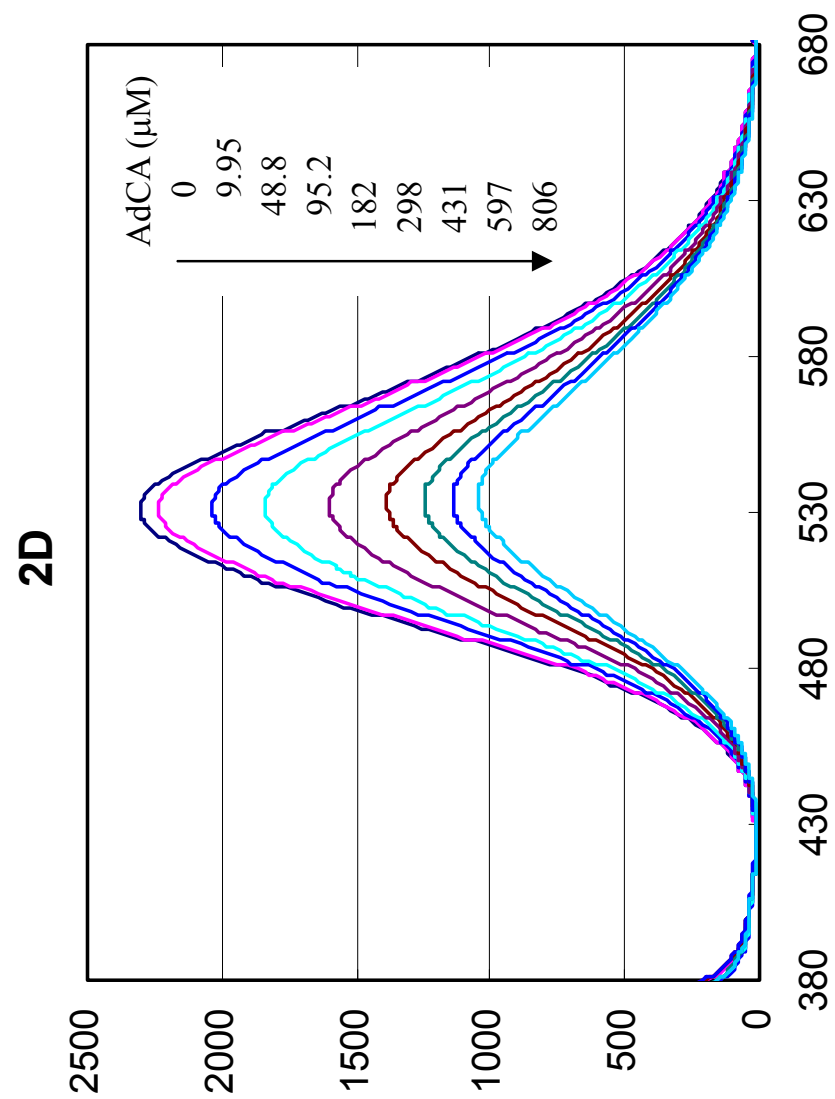

$\stackrel{0}{0}$

$\stackrel{\infty}{+3}$

ঙ্ণ

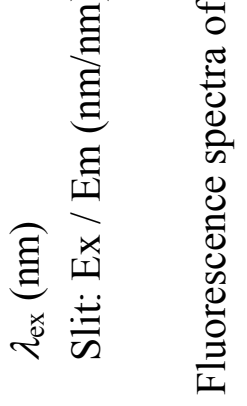



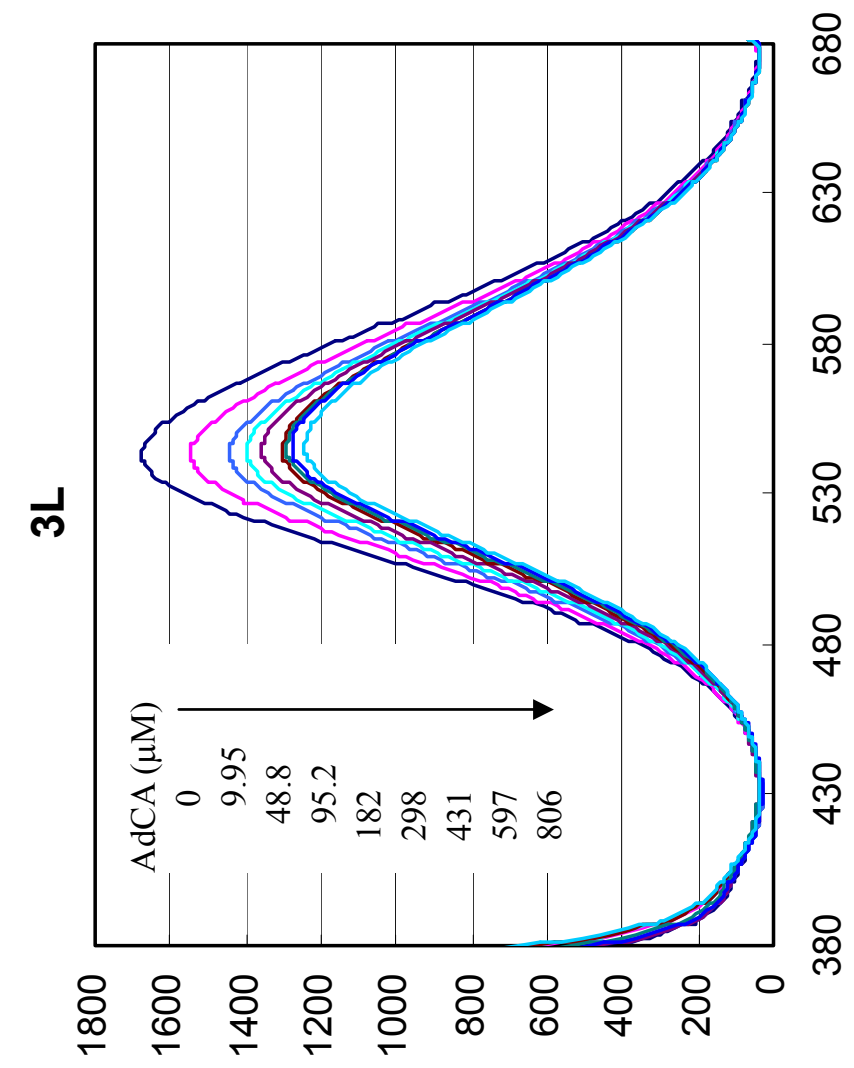

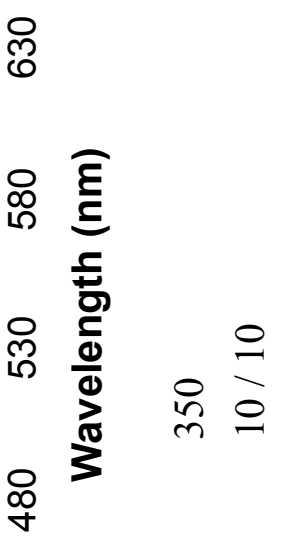

$\frac{\pi}{0}$

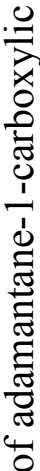

$\stackrel{\%}{\square}$

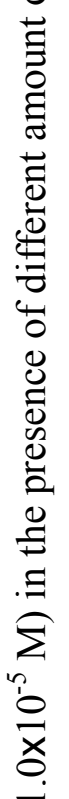

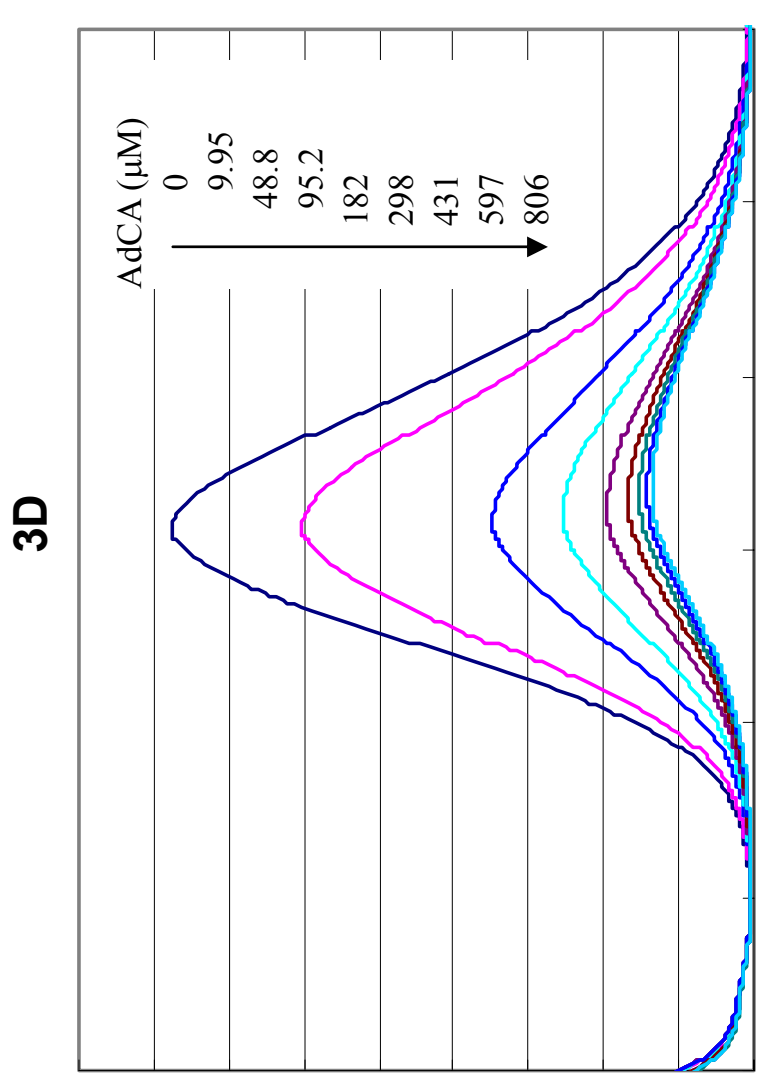

$\stackrel{8}{0}$

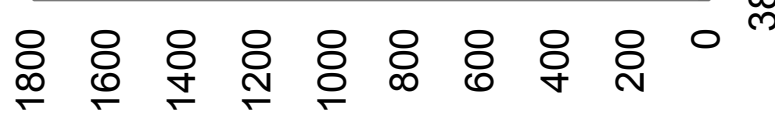

